\title{
Efficient Energy Management System or the technologies of carbon storage and sequestration as aviable alternative to reduce $\mathrm{CO}_{2}$ emissions in the short term
}

\author{
Tirso Lorenzo Reyes Carvajal ${ }^{\mathrm{a}}$, Candido Quintana Pérez ${ }^{\mathrm{b}}$, Ana Emilia Diniz Guedes \\ ${ }^{a}$ Dr., Instituto de Tecnologia e Educação Galileo da Amazônia (ITEGAM) . Brasil. (tirsolrca@ gmail.com) \\ ${ }^{b}$ Dr., Instituto Tecnológico de Santo Domingo. Republica Dominicana (INTEC). (candido.quintana@intec.edu.do) \\ ${ }^{c}$ M.Sc., Universidade do Estado do Amazonas.Brasil (EST/UEA). (aedsguedes@gmail.com)
}

\begin{abstract}
In the present work the results of applying a methodology that complements the focus of many other existing methods of energy management supported the use of statistical and mathematical tools that diagnosis by the energy, thermal and electrical calculations allow appraise the situation in a productive entity or services, moreover, shows a critical analysis comparing to apply a hit management system and storage technology carbon as a means to reduce the negative impact the use of hydrocarbons.
\end{abstract}

Keywords: energy management, carbon storage.

\section{Sistema de Gestão eficiente da energia, ou as tecnologias de sequestro e armazenamento de carbono como uma alternativa viável para reduzir as emissões de $\mathrm{CO}_{2}$ no curto prazo}

\begin{abstract}
RESUMO
No trabalho apresentam os resultados de aplicação de uma metodologia que complementa o enfoque de muitos outros métodos existentes de gestão energética apoiado no uso de ferramentas estatístico-matemática que junto ao diagnostico energético, cálculos térmicos e elétricos permitem valorar a situação de uma entidade produtiva ou dos serviços, além disso, se mostra uma análise crítica de comparação entre a aplicação de um acertado sistema de gestão e a tecnologia de armazenamento de carbono como via para diminuir os impactos negativos do uso dos hidrocarbonetos.
\end{abstract}

Palavras chave: Gestão energética, armazenamento de carbono.

\section{INTRODUÇÃ̃O}

Um programa de Eficiência Energética traz muitos benefícios não somente onde aplicado, mas também em um contexto mais amplo, como diminuição da necessidade de expansão do setor energético, redução de custos em energia em instituições e principalmente contribui com a minimização de impactos ambientais causados pelos gases de efeito estufa lançados à atmosfera.

Um sistema de gestão energética (SGE) visa ter conhecimento de todo o fluxo de energia em uma instituição; verificar influências, possíveis pontos a serem melhorados e acima de tudo ter controle sobre o sistema aplicando ações corretivas.
Em muitos casos, um sistema de gestão de energia é mais eficaz do que programa de eficiência energética devido a sua atuação ser mais ampla e duradoura. Ela demanda um custo inicial relativamente baixo em vista dos retornos vindos de forma de eficiência energética nos diferentes processos [1].

A organização deverá rever e avaliar periodicamente o seu sistema de gestão da energia para identificar oportunidades de melhoria e promover a sua implementação. O ritmo de progressão, a extensão e a duração do processo de melhoria continua são determinados pela própria organização através de: Melhorar a eficiência energética dos seus processos de forma sistemática, estabelecer, programar, manter e melhorar um sistema de gestão energética, assegurar a conformidade com a sua política energética, demonstrar esta conformidade a outros, mediante a certificação do seu sistema de gestão energética por

ITEGAM - JETIA Vol. 01, No. 01. Março de 2015. Manaus - Amazonas, Brasil. ISSN 2447-0228 (ONLINE). 
Tirso-Reyes, Candido-Perez \& Ana-Guedes/ ITEGAM-JETIA Vol.01, Nº 01, pp.48-54. Março, 2015.

uma organização externa, melhorias comerciais: aumento da competitividade, novos mercados (prioridade nas negociações). Redução de custos: otimização do uso de recursos, diminuição da

intensidade energética (consumo energético/PIB). Prevenção de custos: Ferramenta idônea, implantação de atividades resultantes de auditorias energéticas [2].

\section{REVISÂO BIBLIOGRÁFICA}

Há muitas razões para promover o uso eficiente da energia. Os principais fatores de políticas de eficiência energética são a segurança de fornecimento de energia em longo prazo, a eficiência e a competitividade das economias nacionais, e as preocupações ambientais, incluindo o aquecimento global. Particularmente nos países em desenvolvimento, aumento da eficiência energética também contribui para resolver as restrições ao investimento no sector do abastecimento de energia. E de um modo geral, o uso mais eficiente da energia contribui para a igualdade, melhores condições de vida, menores custos de energia e um melhor acesso aos serviços energéticos. $\mathrm{O}$ enorme potencial para o aumento da eficiência energética em todas as fases da produção e uso de energia é amplamente reconhecido, mas percebendo esse potencial ainda é um desafio [3].

A necessidade de reduzir as emissões de gases de efeito estufa (GEEs) e, assim, mitigar os impactos do aquecimento global é uma necessidade urgente e confirmada desde a apresentação dos relatórios do Painel Intergovernamental de Mudanças Climáticas (IPCC, sigla em inglês) da ONU, ao longo dos últimos meses. Segundo a organização não-governamental WWF, o mundo tem apenas cinco anos para que os governos dos países reformulem as suas políticas direcionadas ao fenômeno já inevitável - das mudanças climáticas.

Nesse contexto, a WWF (World Wide Fund for Nature) aposta na viabilidade das tecnologias e das fontes de energia sustentáveis disponíveis para reverter o quadro até 2050. Em seu estudo, a ONG apresenta uma combinação de soluções para atingir o crescimento energético e que consiga atender a demanda.

O curto prazo de cinco anos seria o tempo necessário para o desenvolvimento e a implementação total do uso prático dessas tecnologias. O efeito delas, por outro lado, só será sentido ao longo das próximas décadas. "Se esperarmos mais do que isso, talvez seja tarde demais para iniciarmos este processo de transição sustentável capaz de impedir um aquecimento global maior que $2^{\circ} \mathrm{C}$ ", afirma Denise Hamú, secretária-geral do WWFBrasil. O relatório indica que atrasos tornam a transição mais cara e difícil, além de aumentarem os riscos de fracasso. "Também é fundamental que eles [governos] planejem as dimensões sociais e econômicas desta transição para minimizar os impactos negativos de uma mudança urgente como essa", consideram os pesquisadores do relatório. O documento aponta, por exemplo, que a bioenergia só poderá atingir toda a sua capacidade se produzida de maneira sustentável [4, 10].

Segundo a WWF, o investimento em eficiência energética deve ser a ação mais imediata, enquanto energias alternativas são desenvolvidas. Entre 2020 e 2050, as demandas crescentes dos serviços energéticos podem ser contempladas apenas com esse esforço.

Para isso, os países desenvolvidos devem repotencializar os seus estoques deficientes ou arcaicos, enquanto os em desenvolvimento devem investir, desde o início, em sistemas melhores. Até 2050, o mundo pode alcançar uma economia de $39 \%$ da demanda projetada de energia. Para isso, a eficiência energética deve atingir os setores industriais e de construção. A redução do uso de veículos e as melhorias de motores são estratégias a serem levadas em conta.

Um outro vilão causador das emissões de GEEs são os desmatamentos. Nesse cenário, os países tropicais são agentes que devem se responsabilizar por controlar as taxas de queimadas e de degradação ambiental de florestas. Segundo o IPCC, a preservação florestal pode reduzir as emissões em até $50 \%$.

A redução de combustíveis fósseis é uma meta óbvia. A WWF propõe o uso do gás natural como substituto do carvão, enquanto outras matrizes menos poluentes ainda estiverem em desenvolvimento. O gás natural emite relativamente menos carbono na atmosfera. O relatório calcula que essa opção atingiria um pico de suprimento em 2023.

Nessa fase de transição, em que ainda há uso considerável de combustíveis fósseis, o sistema de captura e armazenamento de carbono, principalmente das indústrias, colaboraria para estabilizar os níveis de emissões em médio prazo (até 2020).

A captura e armazenamento de Dióxido de Carbono $\left(\mathrm{CO}_{2}\right)$ tem o potencial de reduzir significativamente a quantidade de $\mathrm{CO}_{2}$ liberada na atmosfera. As tecnologias necessárias para separar $\mathrm{o} \quad \mathrm{CO}_{2}$ de outros gases e para sequestrar $\mathrm{CO}_{2}$ são conhecidas. Outros desenvolvimentos são necessários para programar a captura e o armazenamento de $\mathrm{CO}_{2}$ em grande escala.

Ao queimarmos combustíveis fósseis-carvão, petróleo e gás natural, estamos aumentando a concentração de $\mathrm{CO}_{2}$, na atmosfera. A concentração de $\mathrm{CO}_{2}$ atualmente é de 379 partes por milhão. É um número significativamente maior do que em qualquer outro momento dos últimos 600.000 anos. $\mathrm{O}$ consenso entre climatologistas é que a maior concentração de $\mathrm{CO}_{2}$ é a principal causa do aumento na temperatura global.

Uma forma de se reduzir a quantidade de $\mathrm{CO}_{2}$ sendo liberado na atmosfera é a maior utilização de fontes alternativas de energia que não produzem $\mathrm{CO}_{2}$. Estas incluem energia hidrelétrica, eólica, solar, nuclear, geotérmica e maremotriz. Cada uma delas tem suas limitações, e será difícil fazer uma troca rápida de combustíveis fósseis para essas outras fontes. Mas e se o $\mathrm{CO}_{2}$ produzido pela queima de combustíveis fósseis não chegar à atmosfera? Em vez de permitir que o $\mathrm{CO}_{2}$ suba pela chaminé e chegue até o ar, podemos capturá-lo e colocá-lo em algum lugar? Isso é possível?

Sim, é possível. O processo é chamado de captura e armazenamento de $\mathrm{CO}_{2}$. Ele tem sido realizado em pequena escala atualmente. Isso tem o potencial de fazer uma diferença considerável na quantidade de $\mathrm{CO}_{2}$ que liberamos na atmosfera. Como o nome sugere, há duas fases no processo. O primeiro desafio é capturar o $\mathrm{CO}_{2}$ em vez de deixá-lo subir pela chaminé. 
Tirso-Reyes, Candido-Perez \& Ana-Guedes/ ITEGAM-JETIA Vol.01, Nº 01, pp.48-54. Março, 2015.

Então ele precisa ser armazenado ou "seqüestrado" de forma segura por um longo tempo. A idéia de seqüestrar o $\mathrm{CO}_{2}$ para reduzir a quantidade que entra na atmosfera é relativamente nova.

¿ Porque o incremento da eficiência energética sobre a base da aplicação de um sistema de gestão energética é a melhor via para a diminuição das emissões de $\mathrm{CO}_{2}$ no setor industrial e dos serviços acorto prazo?

1. Há muita especulação sobre o potencial e tecnologias de sequestro e armazenamento de carbono (SAC) como uma solução para a mitigação de mudanças climáticas.

2. O SAC não será seriamente implementado antes de 2020 e provavelmente não se tornará comercialmente viável como uma opção de mitigação eficiente até 2030. O que é SAC?

Há três diferentes métodos de sequestro ou captura de $\mathrm{CO}_{2}$ : 'précombustão', 'pós-combustão', e 'combustão oxi-combustível'

\section{Pré-combustão:}

* Na captura por 'pré-combustão', o $\mathrm{CO}_{2}$ é removido antes da combustão e então armazenado. No caso do carvão, isto pode ser feito através da gasificação (processo de conversão de carvão de estado sólido a gás, produzindo monóxido de carbono, hidrogênio, metano e nitrogênio)

\section{Pós-combustão:}

* As técnicas de pós-combustão são bem conhecidas no setor energético como prática padrão, por exemplo, para a remoção de enxofre de gás de combustão produzido em usinas de carvão, que normalmente contém até $14 \%$ de $\mathrm{CO}_{2} . \mathrm{O} \mathrm{CO}_{2}$ deve ser separado, por exemplo, por absorção (química ou física), criogenia, e tecnologias de membrana e então ser concentrado, secado e pressurizado para transporte.

\section{Combustão oxi-combustível:}

* Em processos de 'combustão oxi-combustível', o nitrogênio é removido do ar por uma unidade de separação de ar. Oxigênio é adicionado ao combustível, que o acende, emitindo um gás que consiste basicamente de $\mathrm{CO}_{2}$ e vapor de água, que pode ser condensado, resultando em uma corrente seca de $\mathrm{CO}_{2}$ altamente concentrado, comprimido para o transporte e armazenamento.

\section{Limitações e Preocupações}

O SAC levanta uma série de preocupações financeiras, ambientais e 'de princípios':

* SAC é caro. Aumenta os custos da geração de energia entre $40 \%$ e $80 \%$ em comparação com usinas convencionais, dependendo da localização da usina, do local de armazenamento, e da tecnologia de transporte e sequestro utilizada.
* A tecnologia de SAC reduz a eficiência de usinas. Uma quantidade adicional entre 10 e $40 \%$ de combustível fóssil deve ser queimada quando SAC é usado para conseguir o mesmo resultado energético.

* O SAC produz custos adicionais em longo prazo. A monitoração e a verificação ao longo dos anos são necessárias para garantir a retenção do dióxido de carbono armazenado. Ainda assim, oportunidades de intervenção a fim de impedir ou controlar vazamentos inesperados são provavelmente limitadas.

Usinas existentes e as que serão construídas nos próximos cinco a dez anos, não serão repotenciadas com tecnologia SAC depois desse período. A eficiência de uma usina antiga de $33 \%$ de eficiência seria reduzida à faixa de 20 a 25\%, deixando de ser economicamente viável.

A tecnologia de SAC se tornaria economicamente viável apenas com um forte mercado global de carbono, com preços bem acima da faixa de US\$25 a 30 por tonelada equivalente de carbono.

Tecnologias de energia renovável e eficiência energética estão prontas para serem empregadas agora, tanto em termos tecnológicos quanto de mercado. Políticas climáticas sérias demandariam o emprego destas opções, o mais imediato e abrangente possível.

Tecnologias de captura e armazenamento de carbono podem desempenhar uma função, mas mesmo o relatório do IPCC (Intergovermental Panel on Climate Change) sugere que estas serão mais importantes na segunda metade deste século. O clima não pode esperar.

O valor climático de médio a longo prazo do SAC é baixo. Um estudo do instituto Australiano descobriu que o uso isolado de SAC reduziria as emissões australianas em aproximadamente 9\% em 2030, e emissões acumuladas de 2005 a 2030 em apenas $2.4 \%$.

Um cenário com um aumento moderado de eficiência energética poderia reduzir emissões em 2030 em quantidade parecida, e emissões acumuladas em dobro. Isto seria conseguido a um custo zero ou mesmo negativo.

Riscos Ambientais do SAC

Há uma série de riscos e perguntas associadas ao SAC ainda sendo investigadas:

* Quanto tempo o $\mathrm{CO}_{2}$ deve/pode permanecer seguro debaixo da terra? Milhares, dezenas de milhares de anos? O que acontecerá se o $\mathrm{CO}_{2}$ escapar de volta para o ambiente? Este risco deve ser mantido o mais baixo possível. O risco de vazamentos pode ser mantido baixo se escolhido o melhor local geológico para sítios de armazenamento, se houver tempo disponível para tanto. Só é possível conseguir sítios de armazenamento de alta qualidade e padrões de segurança se não houver urgência em armazenar as grandes quantidades anuais de carbono no futuro. 
Tirso-Reyes, Candido-Perez \& Ana-Guedes/ ITEGAM-JETIA Vol.01, Nº 01, pp.48-54. Março, 2015.

* Sítios de armazenamento devem ser monitorados, mas isto será mais difícil se estes forem submarinos, especialmente nos casos de acidentes; todo o trabalho de resgate dependeria das condições metereológicas. Entretanto, um escoamento lento distante do local da injeção seria muito mais difícil ou quase impossível de se detectar. Os impactos biológicos causados pela liberação do $\mathrm{CO}_{2}$ no ambiente marinho podem ser devastadores. Um vazamento mataria organismos incapazes de fugir, dissolveria escudos de carbonato de moluscos, corais ou plânctons específicos, que são a base da cadeia alimentar marinha, com potencias consequências catastróficas [15].

A aplicação de um sistema de gestão energética que denominamos; Sistema de gestão eficiente da energia que fora desenhado por investigadores das universidades cubanas do Cienfuegos e Las Villas e permitiu conhecer os potenciais de economia de portadores energéticos e as possibilidades de diminuição das emissões de $\mathrm{CO}_{2} \mathrm{~A}$ atmosfera. No presente trabalho se mostram os resultados de sua aplicação em 63 organizações

\section{MATERIAIS E MÉTODOS}

A aplicação de um Sistema de Gestão Energética nos permite determinar onde se produzem as perdas durante a operação das instalações industriais, sua quantificação e as causas que a originaram as vias que conduzem a sua redução ou a eliminação total. As formas de organizar os sistemas de gestão energética na empresa ou centros de serviço são variáveis dependendo da atividade empresarial, os interesses e as capacidades das equipes que encarregadas empreender a tarefa na empresa.

Todos coincidem em que o aumento da eficiência energética é a chave do êxito na empresa ou centro de serviço.

A metodologia a seguir no presente trabalho será a seguinte:

\section{METODOLOGIA DO SGEE [5, 7, 8,9]:}

1. Definição dos portadores energéticos.

2. Estabelecimento da estrutura de consumo da empresa, indústria e /o centro de serviço.

3. Estratificação dos resultados.

4. Determinação das áreas ou equipes maiores consumidores.

5. Identificação dos operários e gerentes que decidem na eficiência energética.
6. Estabelecimento e cálculo de indicadores energéticos. Índices de consumo.

7. Realização de um diagnóstico de 1er nível.

8. Calculo dos potenciais de economia de energia.

9. Estabelecimento de parâmetros de operação eficiente.

10. Realização de controle a gerentes, técnicos e operários.

11. Proposta de projetos de melhora

O sistema se apoia no uso de ferramentas estatística matemática que junto ao diagnóstico, cálculos térmicos e elétricos permitem valorar a situação energética da instituição, destacando-se como muito importante os gráficos de correlação Consumo de energia-Produção que dão a conhecer a quantidade de energia não associada à missão da empresa (perdas), todas isso para as áreas e/ou equipes que representam entre o 75-85\% dos consumos.

\section{RESULTADOS E DISCUSÕES.}

A implementação de um sistema de gestão de energia requer um sistema de informação, além da medição do consumo em cada uma das áreas produtivas, processos e máquinas. $\mathrm{O}$ registro histórico do consumo de energia é uma questão fundamental em qualquer empresa, também e um ponto chave para estabelecer valores normativos de consumo de energia de acordo com as características do processo e também permitir comparações com outras empresas que realizam de forma semelhante a produção. A análise das informações fornece o potencial de redução do consumo e, portanto, redução de emissões de gases de efeito estufa. A seguir se apresenta as informações e a comparação de diferentes cenários de acordo com as toneladas de $\mathrm{CO}_{2}$ deixado de emitir no que respeita à implementação do sistema de gestão de energia.

Tabela 1. Consumo de portadores de energia avaliado em tep

\begin{tabular}{|l|c|c|c|c|}
\hline \multicolumn{1}{|c|}{ Portador } & UM & C. Físico & $*$ FC & Cons. tep \\
\hline Eletricidade & MWh/año & 114511 & 0,375 & 42941,5 \\
\hline Diesel & t/año & 37147,3 & 1,0534 & 39130,9 \\
\hline Fuel Oil & t/año & 7672,42 & 0,993 & 7618,7 \\
\hline Gasolina & t/año & 3411,92 & 1,354 & 4619,7 \\
\hline Lubrificantes & t/año & 991,8 & 1 & 991,8 \\
\hline Lenha & t/año & 1044 & 0,3592 & 375 \\
\hline GLP & t/Año & 215,85 & 1,1631 & 251,1 \\
\hline Grasas lubrif. & t/año & 39,85 & 1 & 39,9 \\
\hline Nafta & t/año & 10,71 & 1,0971 & 11,7 \\
\hline Álcool & t/año & 9,86 & 0,6311 & 6,2 \\
\hline Total & - & - & - & 95986,6 \\
\hline
\end{tabular}

*FC-Fator de conversão.

Tabela 2. Das economias possíveis

\begin{tabular}{|c|c|c|c|}
\hline Portador & Das economias possíveis/Ano & Tep/ano & $\begin{array}{c}\text { Valor US\$/ano } \\
\text { (93,58US\$/Barril) }\end{array}$ \\
\hline ELECTRICIDADE MWh/año & 30963,96 & 11611,49 & 7606222,64 \\
\hline DIESEL t/año & 395,67 & 4209,04 & 2757173,74 \\
\hline FUEL OIL t/año & 579,41 & 573,6 & 375742,41 \\
\hline GASOLINA t/año & 12,5 & 16,9 & 11070,51 \\
\hline LUBRIFICANTES t/año & 13,51 & 13,51 & 8849,86 \\
\hline
\end{tabular}


Tirso-Reyes, Candido-Perez \& Ana-Guedes/ ITEGAM-JETIA Vol.01, Nº 01, pp.48-54. Março, 2015.

\begin{tabular}{|c|c|c|c|}
\hline Total & - & 16424,54 & 10759059,17 \\
\hline
\end{tabular}

Referência: Precio de petróleo WTI, martes 26-08-2014: El petróleo WTI ha operado a 93,58 dólares

el barril. Tomando como referência 7 barriles/ton.

\section{Portador Eletricidade}

Para o portador eletricidade foi tomada como base de cálculo cru nacional com $80 \%$ de carbono em sua composição elementar.

Tendo em conta que a Mcarbono $=12 \mathrm{~kg} / \mathrm{kmol}$ e a Moxígeno $=16 \mathrm{~kg} / \mathrm{kmol}$, então a $\mathrm{M}_{\mathrm{CO} 2}=44 \mathrm{~kg} / \mathrm{kmol}$. Dividindo 44 por 12 resulta que por cada unidade de massa de carbono se formam 3,66 de $\mathrm{CO}_{2}$, tem-se então que por cada tonelada queimada de cru nacional são emitidas 2,93 toneladas de $\mathrm{CO}_{2}$ (Resultado do produto de $3,66^{*} 0,80$ )

Como médio em uso final da energia elétrica se consomem 340-360 gramas de combustível por $\mathrm{kWh}$ influenciado pelas perdidas por transmissão e distribuição em linha, equivalente a um médio de $12 \%$.

De acordo Aos consumos do portador eletricidade pelas empresas analisadas se obtém uma contribuição de $125818,6(42941,5 * 2,93)$ toneladas de $\mathrm{CO}_{2}$ à atmosfera e para o potencial de economia obtida se obtém um valor de $34021,69(11611,5 * 2,93)$ toneladas potenciais de economia em términos de emissão de $\mathrm{CO}_{2}$.

Para outros portadores energéticos se fizeram as seguintes considerações:

\begin{tabular}{|l|c|c|}
\hline $\begin{array}{c}\text { Portador } \\
\text { Energético }\end{array}$ & $\begin{array}{c}\text { \% Da } \\
\text { composição } \\
\text { elementar C }\end{array}$ & $\begin{array}{c}\text { Ton de } \mathbf{C O}_{\mathbf{2}} \text { emitidas } \\
\text { por tonelada queimada }\end{array}$ \\
\hline Diesel & 87 & $3,18(3,66 * 0,87)$ \\
\hline Fuel Oil & 80 & $2,93(3,66 * 0,80)$ \\
\hline Gasolina & 85 & $3,11(3,66 * 0,85)$ \\
\hline GLP & 84 & $3,07(3,66 * 0,84)$ \\
\hline
\end{tabular}

Tabela 3. \% de carbono em sua composição elementar.
Tabela 4. Consumo das empresas analisadas

\begin{tabular}{|l|c|c|}
\hline Portador & $\begin{array}{c}\text { Consumo por } \\
\text { portador no } \\
\text { Ton/año }\end{array}$ & $\begin{array}{c}\text { Emissões de } \mathbf{C O}_{\mathbf{2}} \\
\text { no } \underline{\text { Ton/ano. }}\end{array}$ \\
\hline Diesel & 37147,25 & 118284.3 \\
\hline Fuel oil & 7672,42 & 22480.19 \\
\hline Gasolina & 3411,92 & 10611,07 \\
\hline Electricidad & $42941,5(\mathrm{tcc})$ & 125818,6 \\
\hline GLP & 215,85 & 663.61 \\
\hline Total & & 277861.2 \\
\hline
\end{tabular}

O mecanismo de desenvolvimento limpo do PK financia projetos de redução de emissões do GEE (Gases do efeito estufa) mediante a implementação de sistemas de economia de energia, entre outros.

Adotar até 2015 um preço internacional de pelo menos US\$ 32 (EUR 24) por tonelada de carbono $\left(\mathrm{tco}_{2}\right)$ seria necessário para conter de forma eficaz o aquecimento global, A pesquisa, que tem entre os autores o economista britânico Nicholas Stern, uma autoridade em custos das mudanças climáticas, revisou um modelo amplamente usado para avaliar o risco e descobriu que ele levou a uma enorme subavaliação" do risco.

Isto aumenta a necessidade de fortes cortes nas emissões de gases-estufa, impulsionada por um preço de carbono "entre US\$ 32 e US\$ 103 por tonelada de CO2 $\left(t_{\mathrm{CO} 2}\right)$ em 2015", destacou o estudo, realizado pelo "The Economic Journal".

"No prazo de duas décadas, o preço do carbono deveria aumentar em termos reais para US\$ 82 a US\$ 260/tCO2", acrescentou. [17, 18].

Tabela 5. Economias potenciais de portadores energéticos no Ton/ano.

\begin{tabular}{|l|c|c|}
\hline \multicolumn{1}{|c|}{ Portador } & $\begin{array}{c}\text { Economias potenciais de } \\
\text { portadores energéticos no } \\
\text { Ton/año. }\end{array}$ & $\begin{array}{c}\text { Potencial de seqüestro } \\
\text { de CO } \mathbf{C O}_{2} / \mathbf{a n ̃ o}\end{array}$ \\
\hline Diesel & 3995,67 & $12723,01(3995,67 * 3,18)$ \\
\hline Fuel oil & 579,4 & $1697,67(579,4 * 2,93)$ \\
\hline Gasolina & 12,5 & $38,88(12,5 * 3,11)$ \\
\hline Eletricidade & $11611,49(\mathrm{tcc})$ & $34021,69(11611,49 * 2,93)$ \\
\hline GLP & ----- & ------ \\
\hline Total & & 48365,11 \\
\hline
\end{tabular}


Tirso-Reyes, Candido-Perez \& Ana-Guedes/ ITEGAM-JETIA Vol.01, N ${ }^{o}$ 01, pp.48-54. Março, 2015.

Tabela 6. Cenários analisados

\begin{tabular}{|c|c|c|}
\hline \multicolumn{3}{|c|}{ Primeiro Cenário } \\
\hline Portadores & Potencial de seqüestro de $\mathrm{CO}_{2} /$ año & $\begin{array}{c}1^{\circ} \text { Cenário para US\$ } 32 \text { por Ton. } \\
\text { deixada de emitir }\end{array}$ \\
\hline \multirow{2}{*}{$\begin{array}{c}\text { Diesel, fuel oil, gasolina, } \\
\text { eletricidade }\end{array}$} & Total & Total \\
\hline & 48481,25 & US\$ 151400.00 \\
\hline \multicolumn{3}{|c|}{ Segundo Cenário } \\
\hline Portadores & Potencial de sequestro de $\mathrm{CO}_{2} /$ ano & $\begin{array}{c}2 \text { Cenário para US\$ } 52 \text { por Ton. } \\
\text { deixada de emitir }\end{array}$ \\
\hline \multirow{2}{*}{$\begin{array}{l}\text { Diesel, fuel oil, gasolina, } \\
\text { eletricidade }\end{array}$} & Total & Total \\
\hline & 48481,25 & US\$ 2521025.00 \\
\hline \multicolumn{3}{|c|}{ Terceiro Cenário } \\
\hline \multirow[t]{2}{*}{ Portadores } & Potencial de seqüiestro de $\mathrm{CO}_{2} /$ año & $\begin{array}{c}3 \text { Cenário para US\$ } 72 \text { USD por } \\
\text { Ton. deixada de emitir }\end{array}$ \\
\hline & Total & Total \\
\hline $\begin{array}{c}\text { Diesel, fuel oil, gasolina, } \\
\text { eletricidade }\end{array}$ & 48481,25 & US\$ 3490650.00 \\
\hline \multicolumn{3}{|c|}{ Quarto Cenário } \\
\hline \multirow[t]{2}{*}{ Portadores } & Potencial de seqüestro de $\mathrm{CO}_{2} / \mathrm{año}$ & $\begin{array}{c}4^{\circ} \text { Cenário para US\$ } 92 \text { USD por } \\
\text { Ton. deixada de emitir } \\
\end{array}$ \\
\hline & Total & Total \\
\hline $\begin{array}{c}\text { Diesel, fuel oil, gasolina, } \\
\text { eletricidade }\end{array}$ & 48481,25 & US\$ 4460275.00 \\
\hline
\end{tabular}

\section{CONCLUSÕES}

A implementação de medidas de economia permitiriam deixar de emitir $\grave{A}$ atmosfera 48365,11 toneladas de $\mathrm{CO}_{2}$ o qual resulta ser uma importante contribuição á redução do efeito estufa, com a conseguinte mitigação da mudança climática de origem antropogênico no caso estudado

As toneladas de $\mathrm{CO}_{2}$ se fossem negociadas no MDL (Mecanismo de Desenvolvimento Limpo) do PK representariam um benefício econômico de entre um US\$ 151 400.00e US\$ 4460275.00 de acordo ao cenário tirado de referência, fundo o qual poderia ser investido em projetos de Incremento da eficiência energética ou educação ambiental.

Determinação do potencial de economia energética nas empresas para entrar no MDL do Protocolo de Quioto a partir da aplicação de um SGEE não tem precedentes na literatura, com uma contribuição deste trabalho.

O SAC pode ser uma opção no futuro quando todas as perguntas tiverem sido respondidas e os problemas resolvidos, entretanto, agora alcançar maiores níveis de eficiência energética é a solução, mas viável.

A importância de alcançar níveis mais elevados de eficiência energética não se limita aos problemas de poluição ambiental, mas também tem grande importância nos custos associados com a produção ou serviços.

"Somente se nós entendemos o significado do uso eficiente de energia, podemos desfrutar de um futuro mais prazenteiro".

\section{REFERÊNCIAS BIBLIOGRÁFICAS}


Tirso-Reyes, Candido-Perez \& Ana-Guedes/ ITEGAM-JETIA Vol.01, Nº 01, pp.48-54. Março, 2015.

[1] Fernando Frozza.J, Jean-Marc Stephane Lafay, Vitor Baldin, Filipe Marangoni. Metodologia de Implantação de um sistema de Gestão de Energia Utilizando ABNT NBR ISO 50001. Congresso nacional de excelência em gestão. ISSN 1984-9354. 08 e 09 de junho de 2012.

[2] Norma ISO 50001:2011. "Energy management systems.

[3] Augusto Horta L. Indicadores de políticas públicas en materia de eficiencia energética en America latina y el Caribe. Naciones Unidas, Mayo 2010.

[4] IPCC climate Change 2013: The Physical Science Basis. 2013.

[5] Barney L. Capehart, Wayne C. Turner, William J. Kennedy. Guide to Energy Management: International Version. Fifth Edition. Published by The Fairmont Press, Inc. 2008.

[6] G. J. Levermore. 2000. Building Energy Management Systems: Applications to Low-Energy HVAC and Natural Ventilation Controle. Published by Taylor \& Francis.

[7] Reyes Carvajal T.L. Coletivo de autores. 2007. Caracterização energética do setor empresarial na provincial Villa Clara. XV Fórum Nacional de Ciência e Técnica. Dpto. Energia. Universidad Central de las Villas. Cuba.

[8] Borroto Nordelo A. Coletivo de autores. 2002. Gestão Energética Empresarial. CEEMA. Universidade do Cienfuegos. Cuba.

[9] Campos Avella J.C. 2008. Caracterização energética. HYPERLINK"http://ecodesarrollo.cl"

http://ecodesarrollo.cl.

[10] Juan Carlos Brandao 2006. Subdiretor de Meio ambiente da Europa e Latino América. ENDESA. Aciones de eficiência energética e mudança climática no Europa. POWER MEX CLEAN Energy \& EFFICIENCY 2006. XII Seminário de Economia de Energia, Cogeneración e Energia Renovável.
[11] Eficiência Energética: Estudo Mundial Indicadores, Políticas, Avaliação. Relatório do Conselho Mundial da Energia em colaboração com o ADEME Julho 2004.

[12] IDÉIA. 2005. Eficiência energética e energias renováveis. Espanha.

[13] COMISSÃO DAS COMUNIDADES EUROPÉIAS. 22.6.2005. LIVRO VERDE sobre a eficiência energética ou como fazer mais com menos. Bruxelas.

[14]http://www.alice.cnptia.embrapa.br/handle/doc/37311 1.

[15]http://www.greenpeace.org/brasil/pt/Noticias/revoluo-energetica-mostra-o-2/sequeestro-e-armazenamento-de-c/.

[15]http://www.greenpeace.org/brasil/pt/O-quefazemos/Clima-e-Energia/.

[16]http://www.ecodesenvolvimento.org/posts/2014/nicho las-stern-os-riscos-sao-ainda-maiores-do-que?tag=clima.

[17] Simon Dietz and Nicholas Stern. Endogenous growth, convexity of damages and climate risk: how Nordhaus' framework supports deep cuts in carbon emissions, June 2014 Centre for Climate Change Economics and Policy Working Paper No. 180. Grantham Research Institute on Climate Change and the Environment Working Paper No. 159. 